\title{
VLBI observations of 21 extragalactic radio sources
}

\author{
Search for superluminal sources in the mid-southern hemisphere \\ X.Y. Hong ${ }^{1}$, T. Venturi ${ }^{2}$, T.S. Wan ${ }^{1}$, D.R. Jiang ${ }^{1}$, Z.-Q. Shen ${ }^{1}$, X. Liu $^{3}$, G. Nicolson ${ }^{4}$, and G. Umana ${ }^{5}$ \\ 1 Shanghai Astronomical Observatory, Chinese Academy of Sciences, China \\ 2 Istituto di Radioastronomia del CNR, Via Gobetti 101, 40129 Bologna, Italy \\ 3 Urumqi Astronomical Station, Chinese Academy of Sciences, China \\ ${ }^{4}$ Hartebeesthoek Radio Astronomy Observatory, South Africa \\ ${ }^{5}$ Istituto di Radioastronomia del CNR, Noto, Italy
}

Received March 23; accepted July 28, 1998

\begin{abstract}
We present the results of VLBI observations of 21 extragalactic radio sources carried out with an ad-hoc VLBI array including Shanghai, Urumqi, Noto, and Hartebeesthoek radio telescopes. Eleven sources are imaged, three of them for the first time with VLBI. Correlated flux densities of the remaining ten sources are given. From comparison with data taken at earlier epochs, the apparent superluminal motion is confirmed in four sources (0420-014, 0552+398, 1334-127 and 2345-167) and two more superluminal candidates (1504-166 and 2243-123) are found. The physical parameters of the four superluminal sources are derived.
\end{abstract}

Key words: radio continuum: galaxies - galaxies: active - quasars: general

\section{Introduction}

The study of compact radio sources with Very Long Baseline Interferometry (VLBI) has revealed some intrinsic properties of active galactic nuclei (AGN). In some cases, radio emission variability and structural changes relate to high energy outbursts in X-ray and/or $\gamma$-ray regimes. Current models suggest that the emission in these very different energy ranges is connected by means of Inverse Compton processes. This mechanism considers that the relativistic electrons, producing synchrotron radiation, upscatter low energy photons coming either from the synchrotron emission or from other sources (Maraschi et al. 1992).

$\overline{\text { Send offprint requests to }}$ T. Venturi (tventuri@ira.bo.cnr.it)
The phenomenon of apparent superluminal motion, predicted by the theory and found by the VLBI observations, has been explained in terms of relativistic bulk motion along (or very close to) the line of sight of the emitting plasma in AGN. The understanding of the superluminal radio source phenomenon is important for a number of reasons. On one hand, the study of individual sources, possibly observed in a wide range of frequencies, is crucial to understand the emission mechanisms in various parts of the electromagnetic spectrum. On the other hand, the statistical analysis of large samples of superluminal sources addresses more general issues, such as, for example, intrinsic differences among the classes of optical counterparts of superluminal radio sources and tests on cosmological models (Vermeulen 1996). Statistical studies carried out thus far are based on a sample of $\sim 80$ sources, mostly located in the Northern Hemisphere, and the extension of such the sample is important for improving of the statistics.

Two VLBI imaging surveys were carried out in the mid-southern hemisphere using MK2 VLBI recording system with the SHEVE (Southern Hemisphere VLBI Experiment) array plus Shanghai radio telescope at $5 \mathrm{GHz}$ in November 1992 (Shen et al. 1997) and in May 1993 (Shen et al. 1998). The major aims of the experiments are a) search for new superluminal sources; b) fill the gap in southern VLBI observations; c) search for potential targets for Space VLBI; and d) identify compact radio sources as potential southern calibrators and astrometric reference sources. The observations provided first epoch images of 44 sources, and some superluminal candidates were found.

In order to monitor the most interesting sources found in the two previous surveys, and to get information on those radio sources at low declination which had not been observed with VLBI as yet, a third MK2 VLBI experiment at $5 \mathrm{GHz}$ of sources located in the mid-southern 
hemisphere was planned and carried out, using Noto, Shanghai, Urumqi, and Hartebeesthoek telescopes in October 1995. In this paper we present the results of this third MK2 VLBI experiment. In Sect. 2 we describe the source sample. In Sect. 3 we describe the observations and data reduction. Section 4 is a summary of the results.

Throughout this paper, the values $H_{0}=100 h \mathrm{~km} \mathrm{~s}^{-1}$ $\mathrm{Mpc}^{-1}, q_{0}=0.5$, and $S \propto \nu^{\alpha}$ will be used.

\section{The selected sources}

We selected a sample of sources in the mid-southern hemisphere, with $-30^{\circ} \leq \delta \leq+30^{\circ}$. Since the aims of the present work are to monitor the variability in the milliarcsecond scale radio structure, and to collect more sources in the mid-southern hemisphere to supplement the abovementioned VLBI surveys, we included in our sample both core dominated flat spectrum sources and radio sources which had been suggested as superluminal candidates by Shen et al. (1997 and 1998). Our sample includes:

a) seven sources observed and studied in November 1992 and May 1993, which were chosen for monitoring purposes. These are 0420-014, 1034-293, 1334-127, 1504-166, 1730-130, 2243-123, 2345-167;

b) sources with correlated flux density $S_{2.3 \mathrm{GHz}} \geq 0.5 \mathrm{Jy}$ (Preston et al. 1985) and with flat spectra (i.e. $\alpha \sim-0.5$ ).

20 extragalactic radio sources which met the above criteria are selected for the observation. The source DA193 $(0552+398)$ is chosen as amplitude calibrator. The sample is listed in Table 1: Col. 1 - IAU name; Cols. 2 and 3 - positions for the epoch J2000; Col. 4 - optical identification (Q: Quasar , Bl: Blazar); Col. 5 - redshift (Veron-Cetty \& Veron 1995; Preston et al. 1985); Col. 6 - total flux density at $5 \mathrm{GHz}$ : the values with asterisks were measured at Hartebeesthoek during the experiment and the remaining ones are listed in the Parkes Catalogue (Wright \& Otrupcek 1990).

\section{Observation and data processing}

The observation was carried out in a 52 hour experiment on 28-30 October 1995 at $5 \mathrm{GHz}$ in snapshot mode, with each scan lasting 30 minutes. Most of the scans were primarily distributed among the most interesting sources for imaging ( $5-8$ scans on each source), and a few scans were devoted to the weaker sources and those with unknown milliarcsecond structure, in order to obtain their correlated flux densities for further study.

The VLBI array consisted of five antennas: Shanghai, Urumqi, Noto, Medicina and Hartebeesthoek telescopes. No fringes were detected on the baselines to Medicina. Parameters of the remaining four telescopes are listed in
Table 1. The sources for the $5 \mathrm{GHz}$ VLBI observation

\begin{tabular}{ccrccc}
\hline $\begin{array}{c}\text { Source } \\
(1)\end{array}$ & $\begin{array}{c}\text { RA. } \\
(2)\end{array}$ & $\begin{array}{c}\text { Dec. } \\
(3)\end{array}$ & $\begin{array}{c}\text { ID } \\
(4)\end{array}$ & $\begin{array}{c}z \\
(5)\end{array}$ & $\begin{array}{c}S_{5} \mathrm{GHz} \\
(6)\end{array}$ \\
\hline $0008-264$ & 001101.24 & -261233.3 & $\mathrm{Q}$ & 1.093 & 0.81 \\
$0108-079$ & 011050.02 & -074141.2 & $\mathrm{Q}$ & 1.776 & $0.50^{*}$ \\
$0256+075$ & 025927.07 & 074739.6 & $\mathrm{Bl}$ & 0.893 & $0.51^{*}$ \\
$0338-214$ & 034035.61 & -211931.1 & $\mathrm{Q}$ & 0.048 & $0.99^{*}$ \\
$0420-014$ & 042315.80 & -012033.0 & $\mathrm{Bl}$ & 0.915 & 4.15 \\
$0528-250$ & 053007.97 & -250329.8 & $\mathrm{Q}$ & 2.765 & $0.58^{*}$ \\
$0552+398$ & 055530.81 & 394849.2 & $\mathrm{Q}$ & 2.365 & $5.40^{*}$ \\
$0922+005$ & 092507.82 & 001913.9 & $\mathrm{Q}$ & 1.719 & $0.70^{*}$ \\
$1012+232$ & 101447.06 & 230116.5 & $\mathrm{Q}$ & 0.565 & $0.4^{*}$ \\
$1034-293$ & 103716.08 & -293402.8 & $\mathrm{Bl}$ & 0.312 & 1.51 \\
$1334-127$ & 133739.78 & -125724.6 & $\mathrm{Q}$ & 0.539 & 2.18 \\
$1430-178$ & 143257.69 & -180135.2 & $\mathrm{Q}$ & 2.331 & 0.93 \\
$1504-166$ & 150704.78 & -165230.0 & $\mathrm{Q}$ & 0.876 & 1.96 \\
$1532+016$ & 153452.45 & 013104.2 & $\mathrm{Q}$ & 1.435 & 0.79 \\
$1730-130$ & 173302.70 & -130449.5 & $\mathrm{Bl}$ & 0.902 & 4.10 \\
$2131-021$ & 213410.31 & -015317.3 & $\mathrm{Q}$ & 0.557 & $1.65^{*}$ \\
$2243-123$ & 224618.23 & -120651.2 & $\mathrm{Q}$ & 0.630 & $2.24^{*}$ \\
$2320-035$ & 232331.95 & -031705.0 & $\mathrm{Q}$ & 1.411 & $0.82^{*}$ \\
$2325-150$ & 232747.97 & -144755.7 & $\mathrm{Q}$ & 2.465 & $0.83^{*}$ \\
$2345-167$ & 234802.60 & -163112.0 & $\mathrm{Bl}$ & 0.576 & $2.87^{*}$ \\
$2351-154$ & 235430.20 & -151311.2 & $\mathrm{Q}$ & 2.675 & $1.05^{*}$ \\
\hline
\end{tabular}

Table 2. VLBI telescopes and their characteristics at $5 \mathrm{GHz}$

\begin{tabular}{lccc}
\hline Station & $\begin{array}{c}\text { Diameter } \\
(\mathrm{m})\end{array}$ & $\begin{array}{c}T_{\text {sys }} \\
(\mathrm{K})\end{array}$ & $\begin{array}{c}\text { Sensitivity } \\
(\mathrm{K} / \mathrm{Jy})\end{array}$ \\
$(1)$ & $(2)$ & $(3)$ & $(4)$ \\
\hline Shanghai & 25 & 110 & 0.11 \\
Urumqi & 25 & 300 & 0.10 \\
Noto & 32 & 60 & 0.135 \\
Hartebeesthoek & 26 & 80 & 0.082 \\
\hline
\end{tabular}

Table 2. The data were recorded in MK2 recording system with $1.8 \mathrm{MHz}$ bandwidth in left-hand circular polarization. The correlation was performed in Medicina with the MK2 Block-0 Correlator of the IRA-CNR with $1 \mathrm{~s}$ integration time. Fringe fitting was done with the program PHASOR in the Caltech VLBI package. The station based residual fringe rates and the residual delays were estimated with a coherent integration time of 60 seconds. Data post-processing including editing, amplitude calibration and model fitting was carried out using the Caltech VLBI package. Amplitude calibration was done using the program CAL with the system temperatures and the gain curves of all telescopes.

The hybrid images were produced with the DIFMAP program (Shepherd et al. 1995). A point-source model was used as an initial step for iterative self-calibration procedure for all sources. The sources were initially cleaned and phase self-calibrated using uniform weighting in the $(u, v)$ plane, then after several iterations we switched to natural weighting to reduce the image noise. Eleven sources were successfully imaged (see Figs. 1 to 11). The remaining ten sources were observed only for few scans in order to get information on their correlated flux density, and their $u-v$ 
Table 3. Model description of 11 imaged sources

\begin{tabular}{ccccrccr}
\hline Source & No. & $\begin{array}{c}S \\
(\mathrm{Jy})\end{array}$ & $\begin{array}{c}r \\
(\mathrm{mas})\end{array}$ & $\begin{array}{r}\text { PA } \\
\left({ }^{\circ}\right)\end{array}$ & $\begin{array}{c}\text { Major } \\
(\mathrm{mas})\end{array}$ & Ratio & $\begin{array}{r}\text { pa } \\
(1)\end{array}$ \\
$(2)$ & $(3)$ & $(4)$ & $(5)$ & $(6)$ & $(7)$ & $(8)$ \\
\hline $0420-014$ & 1 & 1.64 & 0.00 & 0.0 & 0.40 & 0.65 & -17.5 \\
& 2 & 0.28 & 1.11 & -140.2 & 0.28 & 0.40 & 122.8 \\
& 3 & 0.36 & 1.64 & 172.4 & 0.99 & 0.49 & 31.4 \\
\hline $0552+398$ & 1 & 4.14 & 0.00 & 0.00 & 0.62 & 0.99 & 89.20 \\
$(\mathrm{DA} 193)$ & 2 & 1.24 & 0.75 & -87.29 & 0.41 & 0.79 & 85.21 \\
\hline $1034-293$ & 1 & 1.14 & 0.00 & 0.0 & 0.54 & 0.47 & -59.4 \\
& 2 & 0.26 & 1.63 & 132.4 & 0.99 & 0.96 & 88.7 \\
\hline $1334-127$ & 1 & 2.89 & 0.00 & 0.0 & 0.47 & 0.45 & 24.1 \\
& 2 & 1.03 & 2.11 & 170.2 & 0.41 & 0.48 & 24.5 \\
\hline $1504-166$ & 1 & 1.21 & 0.00 & 0.0 & 0.42 & 0.46 & 5.0 \\
& 2 & 0.40 & 1.35 & 150.2 & 1.11 & 0.62 & 37.7 \\
\hline $1532+016$ & 1 & 0.65 & 0.00 & 0.0 & 0.60 & 0.40 & -71.1 \\
& 2 & 0.18 & 1.24 & 135.7 & 0.22 & 0.89 & 78.8 \\
\hline $1730-130$ & 1 & 3.39 & 0.00 & 0.0 & 0.44 & 0.67 & -1.5 \\
& 2 & 0.49 & 1.96 & -20.2 & 0.73 & 0.98 & 88.4 \\
\hline $2243-123$ & 1 & 1.06 & 0.00 & 0.0 & 0.37 & 0.79 & -8.3 \\
& 2 & 0.53 & 1.07 & -184.3 & 0.25 & 0.29 & -38.4 \\
\hline $2320-035$ & 1 & 0.53 & 0.00 & 0.0 & 0.22 & 0.31 & 156.4 \\
& 2 & 0.13 & 1.29 & -145.9 & 0.74 & 0.53 & 102.6 \\
& 3 & 0.10 & 3.33 & -131.6 & 0.56 & 0.35 & -44.2 \\
\hline $2345-167$ & 1 & 1.59 & 0.00 & 0.0 & 0.54 & 0.58 & 124.6 \\
& 2 & 0.63 & 3.20 & 124.4 & 0.77 & 0.04 & 131.4 \\
\hline $2351-154$ & 1 & 0.73 & 0.00 & 0.0 & 0.38 & 0.32 & -32.1 \\
\hline & & & & & & &
\end{tabular}

coverage was too poor to obtain images. The size of the restoring beam is shown as cross-hatched ellipse in the lower left corner and reported in the caption of each figure. The typical resolution of our images is about 1 mas, depending on the $(u, v)$ coverage in each case. The lowest contour level in each image is 3 times of the rms noise. The rms noise in the images ranges from 1 to $6 \mathrm{mJy} / \mathrm{beam}$. We used the MODELFIT program in the Caltech VLBI package to measure the positions and flux densities of the components in each image. Due to the small amount of data, the maximum number of Gaussian components used to fit both the closure phases and amplitudes in the calibrated data is limited to three. The results are listed in Table 3. In Table 3, Col. 1 is the IAU source name; Col. 2 is the component label; Col. 3 gives the flux density of each component; Cols. 4 and 5 list the distance and position angle of each component with respect to the strongest one, which we assumed to be at the rest frame; Cols. 6,7 and 8 refer to the the parameters of Gaussian model: the width, the axis ratio and the orientation of the individual component, respectively. We estimate that the uncertainties of the parameters are: $\Delta S= \pm 20 \%, \Delta r= \pm 0.1 \mathrm{mas}$, $\Delta \mathrm{PA}= \pm(5-10)^{\circ}, \Delta$ Major $= \pm 15 \%, \Delta$ Ratio $= \pm 20 \%$, $\Delta \mathrm{PA}= \pm(5-10)^{\circ}$.

\section{Results and discussion}

\subsection{Superluminal sources}

4.1.1. Individual sources

$0420-014$

The core-dominated highly polarized blazar 0420-014 $(z=0.915)$ is known to be active and variable at all wavelengths. It is a ROSAT source (Brinkmann et al. 1994), and has been identified with an EGRET $\gamma$-ray source (Fichtel et al. 1994). At radio frequencies, the morphology of the source shows a typical core-jet structure from milliarcsecond (Krichbaum et al. 1994; Wagner et al. 1995; Shen et al. 1997) to arcsecond scale (Antonucci \& Ulvestad 1985). Simultaneous optical and $\gamma$-ray flares in 1992 were found (Wagner et al. 1995). There is an outburst at millimeter wavelengths in 1992 (Tornikoski et al. 1996).

Our $5 \mathrm{GHz}$ image of $0420-014$ (Fig. 1) shows a bent morphology from position angle (PA) $-140^{\circ}$ to $-187.6^{\circ}$. The jet structure is in agreement with previous results (Krichbaum et al. 1994; Wagner et al. 1995). The data can be fitted with three components. The model fitting parameters we obtained are listed in Table 3. Comparison of our results with those relative to epoch 
1992 (Wagner et al. 1996) shows that components 2 and 3 moved away from the bright component with an

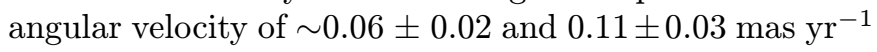
respectively, corresponding to apparent transversal speeds $\beta_{\text {app }}=1.7 \pm 0.6 \mathrm{~h}^{-1}$ and $2.9 \pm 0.8 h^{-1}$. Superluminal motion in the jet component 2 was reported, with a speed $\beta_{\text {app }}=3.9 h^{-1}$, at $8.4 \mathrm{GHz}$ between epochs 1990 and 1992 (Wagner et al. 1995) and at $5 \mathrm{GHz}$ during the period 1986.50 to 1992.88 (Shen et al. 1997).

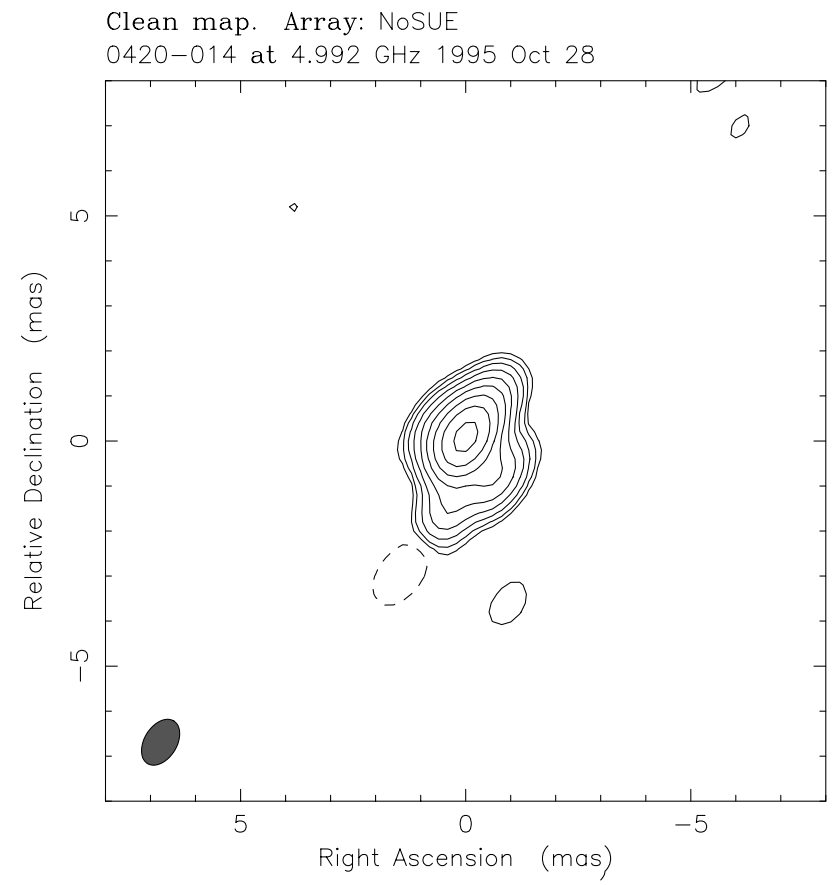

Fig. 1. Image peak: $1.47 \mathrm{Jy} /$ beam. Contours: $1.5 \mathrm{mJy} /$ beam $\times$ $(-3,3,6,12,24,48,96,192,384,768)$. Beam FWHM: $1.11 \times$ 0.722 (mas) at $-31.8^{\circ}, \mathrm{rms}=1.5 \mathrm{mJy} /$ beam

\section{$0552+398$ (DA193)}

DA193 $(z=2.365)$ is one of the most compact radio sources known to date. It is identified with a 18 th magnitude quasar. The source has been observed with VLBI at several frequencies. At $2.3 \mathrm{GHz}$, DA193 was barely resolved with a slight extension in $\mathrm{PA}=90^{\circ}$ (Charlot 1990). At $5 \mathrm{GHz}$, it showed a core-jet structure consisting of two components with a separation of 0.37 mas at $\mathrm{PA}=-80^{\circ}$ at epoch 1981.61 (Spangler et al. 1983). At $8.4 \mathrm{GHz}$, the VLBI image showed an extension at $\mathrm{PA}=-75^{\circ}$ with a separation of 0.52 mas at epoch 1985.37 (Charlot 1990), and at $\mathrm{PA}=-71^{\circ}$ with a separation of 0.7 mas at epoch 1994.52 (Fey et al. 1996). At $10.7 \mathrm{GHz}$, the source showed a core halo structure with the major axis of the halo at $\mathrm{PA}=80^{\circ}$ (Schilizzi et al. 1981).

DA193 was also observed at $5 \mathrm{GHz}$ using a complete track to test VLBA for high dynamic range image. The source is resolved at a resolution of 1.8 mas $\times 1.5$ mas,

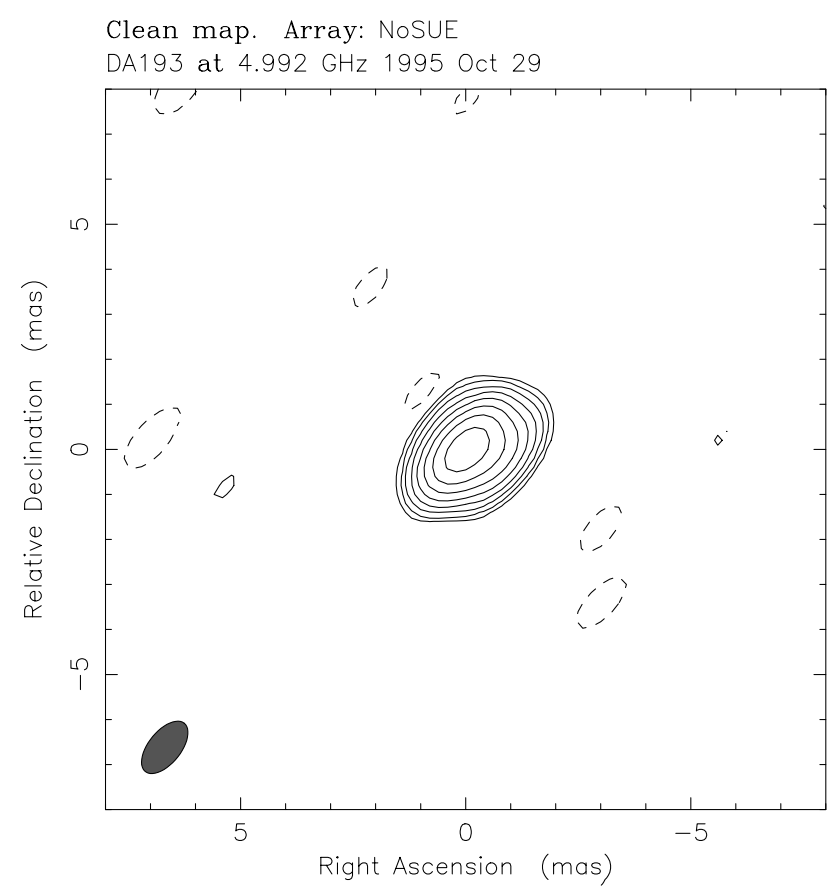

Fig. 2. Image peak: $3.81 \mathrm{Jy} /$ beam. Contours: $6 \mathrm{mJy} /$ beam $\times$ $(-3,3,6,12,24,48,96,192,384)$. Beam FWHM: $1.38 \times 0.719$ (mas) at $-38.9^{\circ}, \mathrm{rms}=6 \mathrm{mJy} /$ beam

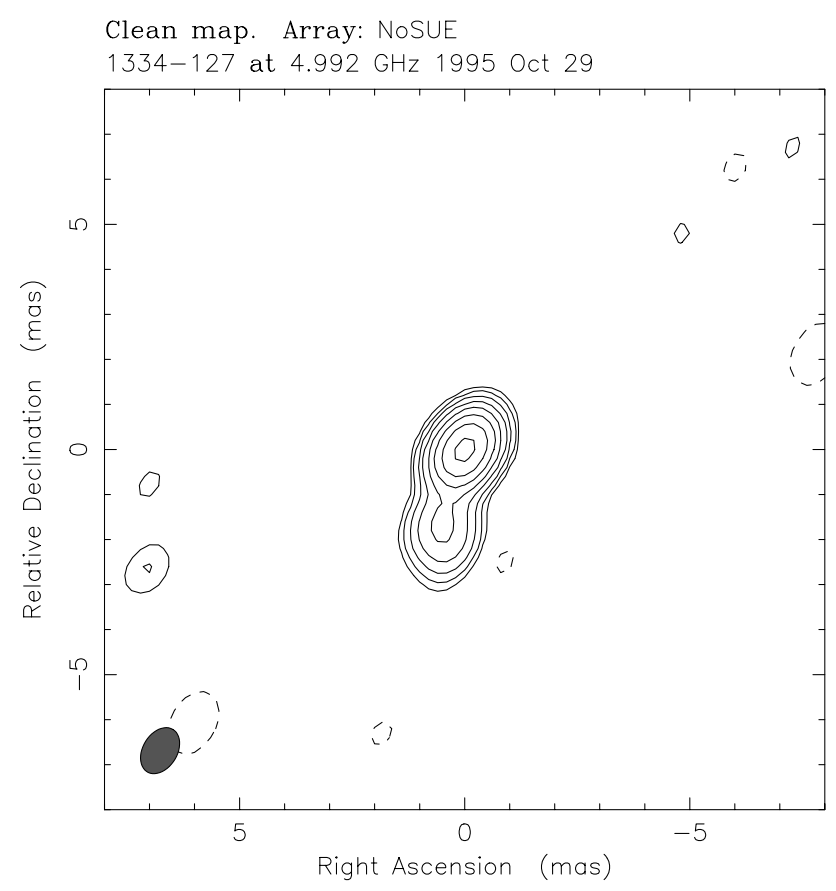

Fig. 3. Image peak: $2.8 \mathrm{Jy} /$ beam. Contours: $6 \mathrm{mJy} /$ beam $\times$ $(-3,3,6,12,24,48,96,192,384)$. Beam FWHM: $1.11 \times$ 0.765 (mas) at $-31.2^{\circ}, \mathrm{rms}=6 \mathrm{mJy} /$ beam 


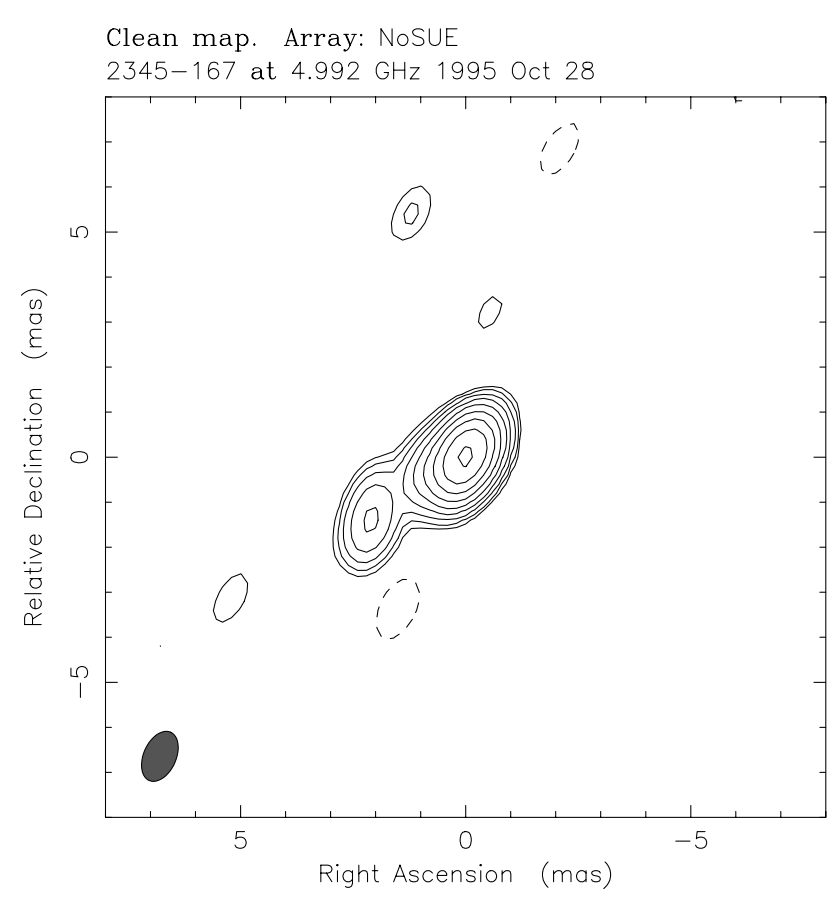

Fig. 4. Image peak: $1.75 \mathrm{Jy} /$ beam. Contours: $2 \mathrm{mJy} /$ beam $\times$ $(-3,3,6,12,24,48,96,192,384,768)$. Beam FWHM: $1.17 \times$ 0.723 (mas) at $-24.1^{\circ}, \mathrm{rms}=2 \mathrm{mJy} /$ beam

and can be fitted by a two elliptical Gaussian component model (Briggs et al. 1994).

Our $5 \mathrm{GHz}$ image of the source is shown in Fig. 2. It can be fitted by two components: a compact strong core component of $4.14 \mathrm{Jy}$ and a jet component of $1.24 \mathrm{Jy}$ with a separation of 0.75 mas in $\mathrm{PA}=-87.3^{\circ}$. Charlot (1990) estimated a proper motion of 0.04 mas $\mathrm{yr}^{-1}$ from the epoch 1981 at $5 \mathrm{GHz}$ to epoch 1985 at $8.4 \mathrm{GHz}$, which corresponds to an apparent transversal speed $\beta_{\text {app }}=1.7 h^{-1}$.

Our model fitting of the source shows a separation of 0.75 mas between the two components (Table 3). By comparing the result with that of Spangler et al. (1983) at the same frequency, an average apparent angular velocity of

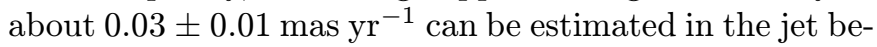
tween epoch 1981.61 and epoch 1995.83, which is same as reported by Bajkova et al. (1996). The proper motion corresponds to a speed of $1.3 \pm 0.4 h^{-1}$ c. No obvious proper motion has been detected at $8.4 \mathrm{GHz}$ by VLBA between July of 1994 and October of 1995 (Fey et al. 1996, 1997), because the time scale is too short to detect it.

\section{4-127}

$1334-127(z=0.539)$ is a highly polarized quasar (Impey \& Tapia 1990). Its VLA image at $1.4 \mathrm{GHz}$ shows a curved jet extending 6.5 arcseconds to the east of the source (Perley 1982). The source was detected by ROSAT, with a flux density of $0.63 \mu \mathrm{Jy}$ at $1.3 \mathrm{KeV}$ (Brinkmann et al. 1994).

VLBI observation carried out at $5 \mathrm{GHz}$ in 1986.9 (Wehrle et al. 1992) indicated that the source was barely resolved. A jet component was located at 1.7 mas from the core in $\mathrm{PA}=-150^{\circ}$ and by comparison with the 1992.88 data, a proper motion of 0.28 mas $\mathrm{yr}^{-1}$ was estimated, which corresponds to a $\beta_{\text {app }}=4.5 h^{-1}$ (Shen et al. 1997). VLBA images show a core-jet structure in $\mathrm{PA} \sim 150^{\circ}$ at $2.3 \mathrm{GHz}, \mathrm{PA} \sim 160^{\circ}$ at $8.4 \mathrm{GHz}$ (Fey et al. 1996), and $\mathrm{PA} \sim 160^{\circ}$ at $15 \mathrm{GHz}$ (Kellermann et al. 1998).

Our image is shown in Fig. 3. The source shows a jet structure extending to south-east $\left(\mathrm{PA} \sim 170^{\circ}\right)$. The parameters of the model fitting are listed in Table 3 . The comparison of our map with the published data at similar resolution indicate that the position angle of the jet component is quite different at different epochs. The reason for such difference is unclear and needs further investigation. Assuming that the jet component at different epochs is the same one, we derived that it moves with an apparent velocity of $\beta_{\mathrm{app}}=2.4 \pm 0.5 \mathrm{~h}^{-1}$ by comparing the results of two epochs (1992.88 and 1995.83) of observation at same frequency.

\section{5-167}

$2345-167(z=0.576)$ is an optically violent variable and a highly polarized blazar. X-ray emission was detected by ROSAT (Brinkmann et al. 1994). It has a complex radio spectrum with a peak around $5 \mathrm{GHz}$, and it is a low frequency variabile source (McAdam and White 1983). VLA observations show a jet of 4.0 arcseconds in $\mathrm{PA}=-130^{\circ}$.

Our image of $2345-167$ shows a clear core-jet structure (Fig. 4). The jet component is located 3.2 mas from the core in $\mathrm{PA}=124^{\circ}$. Comparing our result with that of epoch 1992.88 (Shen et al. 1997), a proper motion of 0.08 \pm 0.03 mas $\mathrm{yr}^{-1}$ can be estimated, which corresponds to a $\beta_{\text {app }}=1.6 \pm 0.7 h^{-1}$. A proper motion of 0.26 mas $\mathrm{yr}^{-1}$, corresponding to an apparent velocity of about $5 h^{-1} \mathrm{c}$, was reported by Shen et al. (1997). Our result suggests that the jet decelerates as the component moves outwards, as observed in other sources. The pc scale structure is almost orthogonal to the arcsecond scale structure, as seen in some radio loud quasars such as 3C 345. A flux density of $0.65 \mathrm{Jy}$ is missing from the map of 2345-167. This may indicate the source has extended emission.

\subsubsection{The physical parameters}

In this sub-section we estimate the physical parameters of the four superluminal sources and report them in Table 4, which contain the following information: in Col. 1 we give the source name; Col. 2 reports the X-ray flux density at $1.3 \mathrm{keV}$ (Brinkmann et al. 1994). No $\mathrm{X}$-ray emission has been detected for DA193; Col. 3 gives the FWHM angular size of the VLBI core, computed as $\theta_{\mathrm{FWHM}}=\sqrt{\theta_{\max } \times \theta_{\min }}$, where $\theta_{\max }$ and $\theta_{\min }$ are respectively the major and minor axis of the VLBI core, as given 
Table 4. Observed and physical parameters of four superluminal sources

\begin{tabular}{ccccccc}
\hline Source & \multicolumn{1}{c}{$S_{\mathrm{x}}$} & $\theta_{\mathrm{FWHM}}$ & $\delta_{\text {eq }}$ & $\delta_{\mathrm{IC}}$ & $\gamma$ & $\phi$ \\
IAU & $\mu \mathrm{Jy}$ & mas & & & & $\left({ }^{\circ}\right)$ \\
$(1)$ & $(2)$ & $(3)$ & $(4)$ & $(5)$ & $(6)$ & $(7)$ \\
\hline $0420-014$ & 0.72 & 0.32 & 9.4 & 7.1 & $6.5 \pm 2.4$ & $5.4 \pm 5$ \\
$\mathrm{DA} 193$ & & 0.62 & 9.8 & $5.0 \pm 2.5$ & $2 \pm 10$ \\
$1334-127$ & 0.63 & 0.32 & 14.4 & 10.3 & $10.4 \pm 3.6$ & $4 \pm 4$ \\
$2345-167$ & 0.21 & 0.41 & 4.3 & 4.7 & $3.5 \pm 1.1$ & $13 \pm 10$ \\
\hline
\end{tabular}

in Table 3. The equipartition Doppler factor $\delta_{\text {eq }}$, the inverse Compton Doppler factor $\delta_{\mathrm{IC}}$, the Lorentz factor $\gamma$ and the viewing angle between the jet axis and the line of sight $\phi$ are computed and listed in Cols. 4, 5, 6 and 7 respectively.

\section{Equipartition Doppler factor $\delta_{\mathrm{eq}}$}

The Doppler factor of the outflow from the compact radio cores can be estimated assuming that the particles and magnetic field are in equipartition (Readhead 1994; Guijosa \& Daly 1996),

$$
\begin{aligned}
\delta_{\mathrm{eq}}= & {\left[\left[10^{3} F(\alpha)\right]^{34}\left\{\left[1-(1+z)^{-1 / 2}\right] / 2 h\right\}^{-2}(1+z)^{15-2 \alpha}\right.} \\
& \left.\times S_{\mathrm{op}}^{16} \theta_{\mathrm{d}}^{-34}\left(\nu_{\mathrm{op}} \times 10^{3}\right)^{-(2 \alpha+35)}\right]^{1 /(13-2 \alpha)}
\end{aligned}
$$

where $F(\alpha)$ is given by Scott \& Readhead (1977), and $\alpha$ (assumed to be -0.75 ) is the spectral index of the radio emission. In particular, $F(-0.75)=3.4 . z$ is the redshift, $S_{\text {op }}$ is the observed peak flux density in Jy, at the observed frequency $\nu_{\mathrm{op}}$ in GHz. The angular diameter $\theta_{\mathrm{d}}$ (in mas) of the core component is greater than the observed angular diameter. Marscher (1987) suggested a correction for this by using $\theta_{\mathrm{d}}=1.8 \theta_{\text {FWHM }}$. Column 4 lists the equipartition Doppler factors (Eq. (1)) computes assuming $h=1$, $S_{\text {op }}=S_{\text {obs }}$, and $\nu_{\text {op }}=\nu_{\text {obs }}$, where $\nu_{\text {obs }}$ is the frequency of our observations and $S_{\text {obs }}$ is the flux density we derived.

\section{Inverse Compton Doppler factors $\delta_{\mathrm{IC}}$}

For comparison we also estimated the Doppler factor $\delta$ with the self-Compton emission in a uniform spherical model (Marscher 1987) by comparing the predicted flux with the observed Self-Compton flux (Ghisellini et al. 1993),

$\delta_{\mathrm{IC}} \approx f(\alpha) S_{\mathrm{m}}\left[\frac{\ln \left(\nu_{\mathrm{b}} / \nu_{\mathrm{m}}\right) \nu_{\mathrm{x}}^{\alpha}}{S_{\mathrm{x}} \theta_{\mathrm{d}}^{6-4 \alpha} \nu_{\mathrm{op}}^{5-3 \alpha}}\right]^{\frac{1}{4-2 \alpha}}(1+z)$,

where $S_{\mathrm{x}}$ is the observed X-ray density (in Jy) at frequency $\nu_{\mathrm{x}}(\mathrm{keV}), \nu_{\mathrm{op}}$ is the frequency at which the radio peak occurs (in $\mathrm{GHz}$ ), and $\nu_{\mathrm{b}}$ is the synchrotron high-frequency cutoff, which we assumed to be $10^{5} \mathrm{GHz}$. Our choice is justified by the fact that our objects are blazars. Furthermore, $f(\alpha) \approx-0.08 \alpha+0.14 ; \alpha$, assumed to be -0.75 , is the spectral index of the radio emission and $\theta_{\mathrm{d}}=1.8 \theta_{\mathrm{FWHM}}$. From the use of the extrapolated value of the flux density (Marscher 1987) we can derive that the flux $S_{\mathrm{m}}$ is about a factor of 2 larger than the observed peak flux density $S_{\text {op }}$. Column 5 in Table 4 lists the $\delta_{\mathrm{IC}}$, computed with Eq. (2). The $\delta_{\mathrm{IC}}$ of $0420-014$ and $1334-127$ is less than its $\delta_{\text {eq }}$. This can be explained assuming that $\delta_{\mathrm{IC}}$ should be considered a lower limit, since part of the X-ray emission may come from different mechanisms rather than Inverse Compton.

\section{Lorentz factor and viewing angle}

In the relativistic beaming model $\beta_{\text {app }}$ is related to the true velocity $v=\beta c$, and the angle to the line of sight $\phi$ (e.g., Pearson \& Zensus 1987),

$\beta_{\mathrm{app}}=\frac{\beta \sin \phi}{1-\beta \cos \phi}$

and the Doppler factor can be written as

$\delta=\gamma^{-1}(1-\beta \cos \phi)^{-1}$,

where $\gamma=1 / \sqrt{1-\beta^{2}}$. On the basis of Eqs. (3) and (4), the Lorentz factor $\gamma$ and the angle to the line of sight $\phi$ can be calculated with known $\beta_{\text {app }}$ and $\delta$. Columns 6 and 7 of Table 4 list the Lorentz factors and viewing angles which were estimated by assuming $h=1$ and $\delta=\delta_{\text {eq }}$.

We can see that these four sources have properties typical of superluminal objects, i.e. high Doppler boosting and small viewing angle between the jet axis and the line of sight.

\subsection{Superluminal candidates}

\section{$1504-166$}

$1504-166(z=0.876)$ is classified as a highly polarized quasar (Impey \& Tapia 1990), and is known to be a low frequency variable source (Padrielli et al. 1986). It was unresolved at $1.4 \mathrm{GHz}$ by VLA (Perley 1982).

Our VLBI image shows a slightly curved core-jet structure to the south-east (Fig. 5). Our model fitting consists of two components, and the parameters are listed in Table 3. The jet position angle is consistent with that derived at $1.7 \mathrm{GHz}$ by Romney et al. (1984). It seems that the jet component is bending clockwise, and closer to the the direction of the jet component at $1.7 \mathrm{GHz}$ (Padrielli et al. 1986). The $5 \mathrm{GHz}$ VLBI image obtained by Shen et al. (1997) at epoch 1992.83 consists of three components, i.e. a compact core with a flux density of $1.3 \mathrm{Jy}$ and a diameter of 0.5 mas (component 1), a $0.6 \mathrm{Jy}$ jet at 1.12 mas from core in $\mathrm{PA}=-156^{\circ}$ (component 2 ) and a $0.3 \mathrm{Jy}$ component at 0.8 mas from core in $\mathrm{PA}=161^{\circ}$ (component 3). Assuming the jet component 2 in our image is the same as the second component at epoch 1992 (Shen et al. 1997), we can estimate a proper motion of 0.08 mas $\mathrm{yr}^{-1}$, which corresponds to $\beta_{\text {app }}=1.5 h^{-1}$. Therefore we suggest that $1504-166$ is a superluminal candidate. 


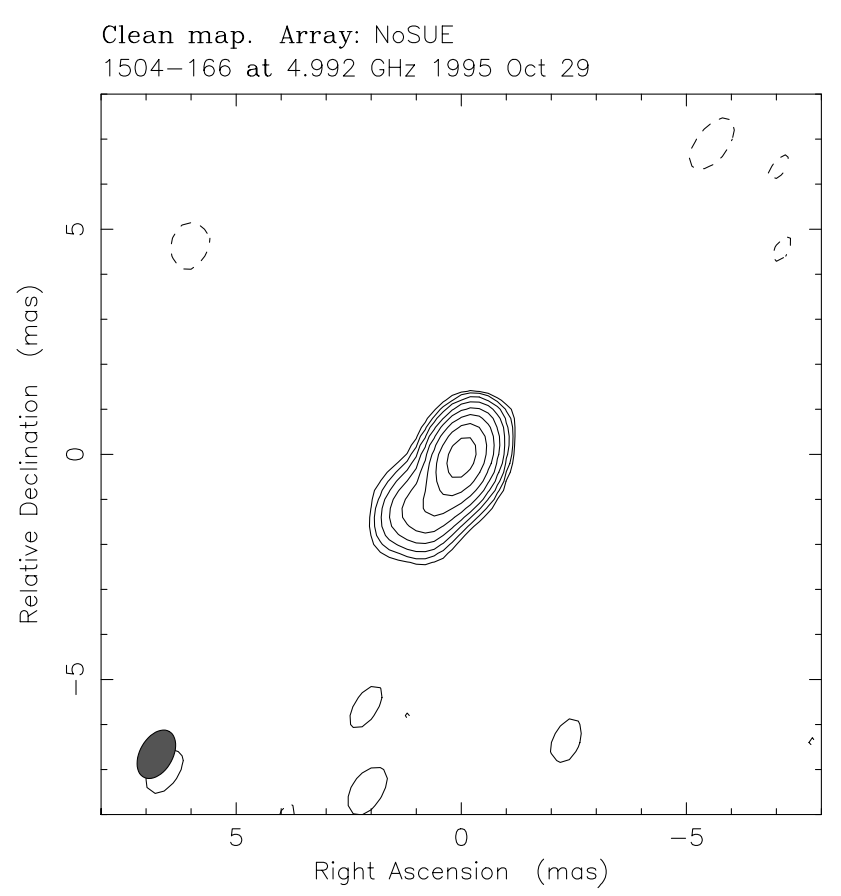

Fig. 5. Image peak: $1.13 \mathrm{Jy} /$ beam. Contours: $2 \mathrm{mJy} /$ beam $\times$ $(-3,3,6,12,24,48,96,192,384)$. Beam FWHM: $1.16 \times 0.733$ (mas) at $-29.3^{\circ}, \mathrm{rms}=2 \mathrm{mJy} /$ beam

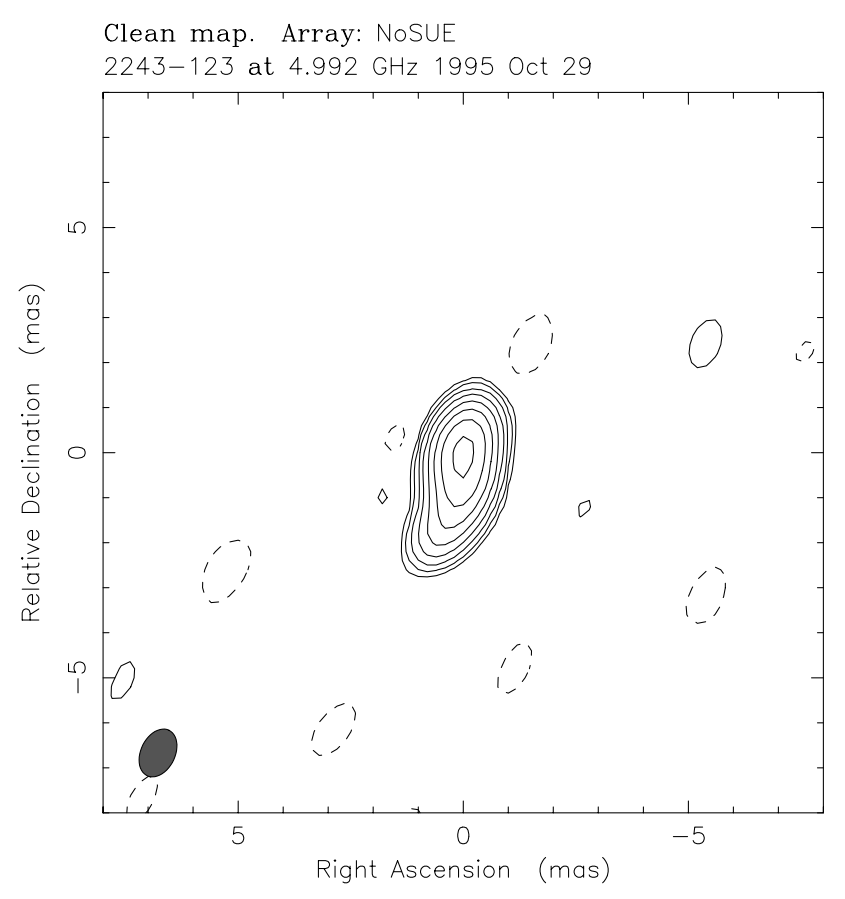

Fig. 6. Image peak: $0.95 \mathrm{Jy} /$ beam. Contours: $2 \mathrm{mJy} /$ beam $\times$ $(-3,3,6,12,24,48,96,192,384)$. Beam FWHM: $1.12 \times$ 0.76 (mas) at $-25.7^{\circ}, \mathrm{rms}=2 \mathrm{mJy} /$ beam

\section{$2243-123$}

2243-123 $(z=0.630)$ is a highly polarized quasar. Emission at X-ray and $\gamma$-ray energies was detected (Maisack et al. 1994, Fichtel et al. 1994). The VLA image shows an unresolved core and an extended component at $4^{\prime \prime}$ from the core in $\mathrm{PA}=40^{\circ}$ (Morganti et al. 1993; Browne et al. 1986; Perley 1982).

Our new image (Fig. 6) shows a core-jet structure, with a jet component located at 1.07 mas to the south of the core. A VLBI image of the source at $5 \mathrm{GHz}$ at epoch 1993 shows a compact central core elongated in the north-south direction (Shen et al. 1998). The total flux density monitoring at millimeter wavelength obtained by single dish observations, shows a peak at 1992.90 (Tornikoski et al. 1996). If we assume that the jet component was ejected as a consequence of that outburst, the detected proper motion of the jet would be 0.40 mas $\mathrm{yr}^{-1}$, which corresponds to $\beta_{\text {app }}=4.2 h^{-1}$. So, 2243-123 may be considered as a new superluminal source candidate.

\subsection{First epoch VLBI images of three sources}

\section{$1532+016$}

The source $1532+016$ is identified with a 19.8 magnitude quasar (McEwan et al. 1975) with emission lines, and is located at a redshift $z=1.435$. X-ray and infrared emission of the source were detected (Impey 1988). VLA observations show that the source has a secondary component at $1.4^{\prime \prime}$ from the core in $\mathrm{PA}=45^{\circ}$ (Perley 1982). A correlated flux density of $0.67 \mathrm{Jy}$ at $8.4 \mathrm{GHz}$ with VLBI resolution for this source was reported by Morabito et al. 1986 .

We obtained the first VLBI image of $1532+016$ (Fig. 7), which shows a structure extended in $\mathrm{PA}=135^{\circ}$. The data can be fitted by two components. The model parameters are listed in Table 3 . We point out that the milliarcsecond scale structure is orthogonal to the arcsecond scale structure, as often seen in radio loud quasars.

\section{0-035}

2320-035 is a 19th magnitude quasar at $z=1.411$ (Punsly 1995). VLA observations show that the source is unresolved on the arcsecond scale. In Fig. 8 we present the first epoch $5 \mathrm{GHz}$ VLBI image of the source. The image shows a core-jet structure oriented to south-west. The data can be fitted by three components (see Table 3).

\section{1-154}

The source $2351-154(z=2.675)$ is associated with a 17th magnitude quasar (Hewitt \& Burbridge 1993). Its X-ray emission was detected by Brinkmann et al. (1994). The VLA image of the source at $1.6 \mathrm{GHz}$ shows a deformed core, elongated to northeast (Neff \& Hutchings 1990), which is interpreted as a jet seen nearly end on (Punsly 1995). 


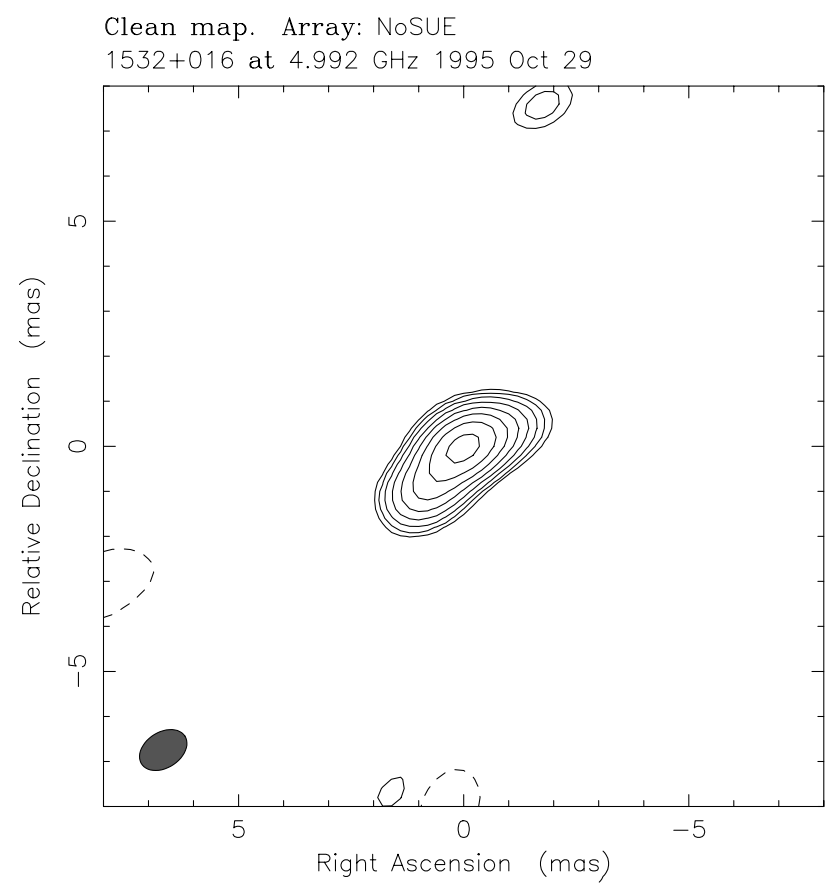

Fig. 7. Image peak: $0.51 \mathrm{Jy} /$ beam. Contours: $1 \mathrm{mJy} /$ beam $\times$ $(-3,3,6,12,24,48,96,192,384)$. Beam FWHM: $1.14 \times 0.79$ (mas) at $-57.2^{\circ}, \mathrm{rms}=1 \mathrm{mJy} /$ beam

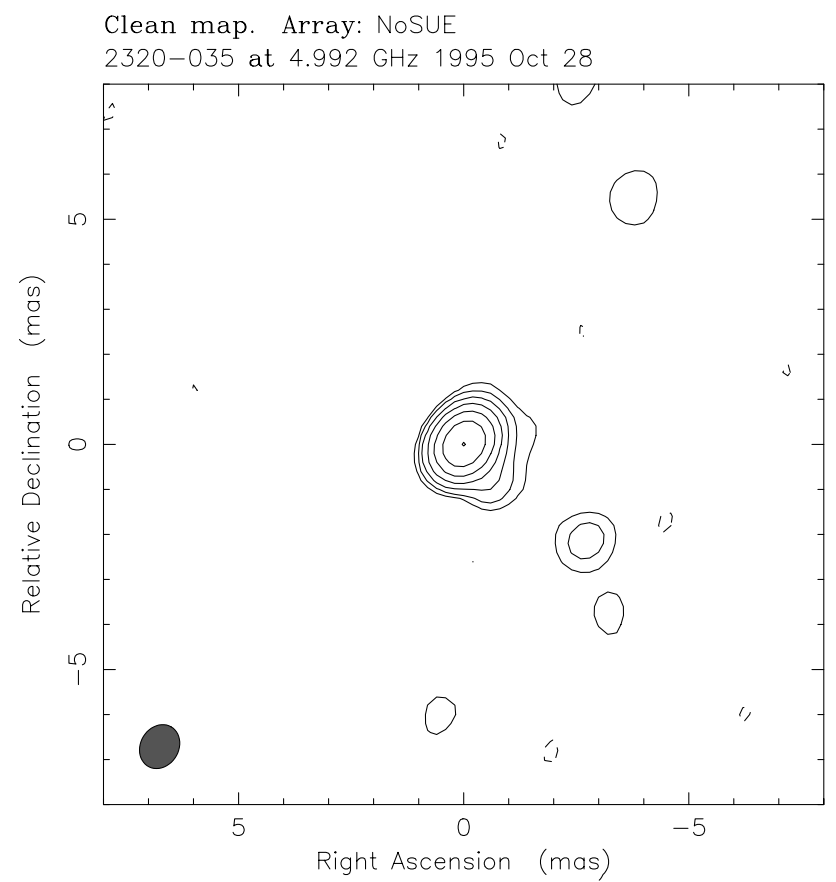

Fig. 8. Image peak: $0.49 \mathrm{Jy} /$ beam. Contours: $2.5 \mathrm{mJy} /$ beam $\times$ $(-3,3,6,12,24,48,96,192)$. Beam FWHM: $1.02 \times 0.85$ (mas) at $-30.1^{\circ}, \mathrm{rms}=2.5 \mathrm{mJy} /$ beam

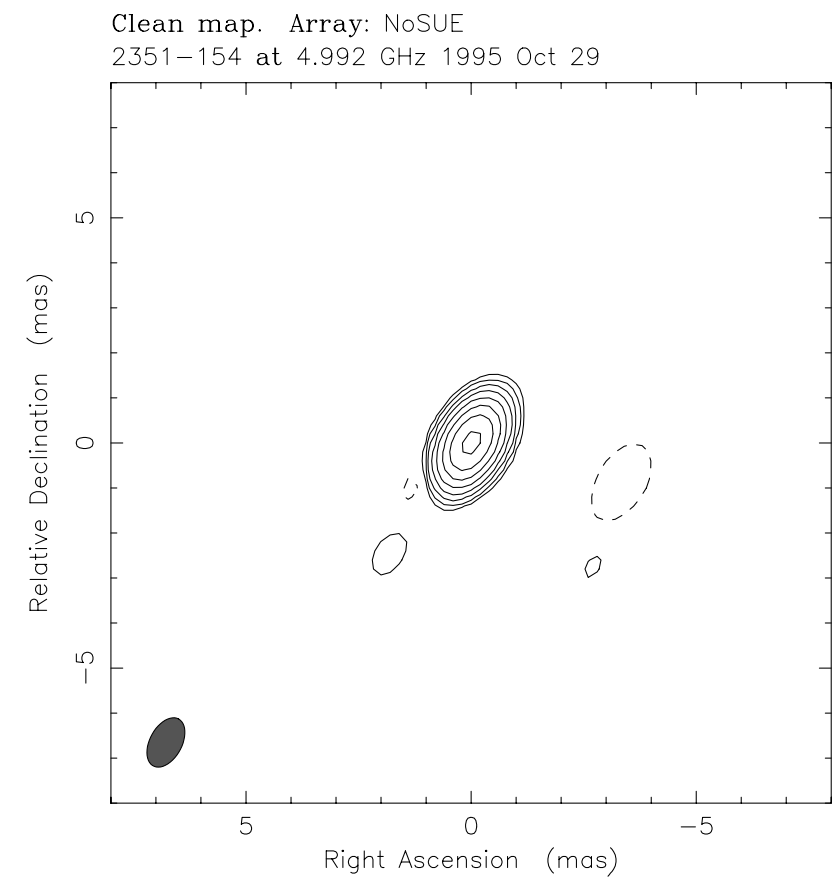

Fig. 9. Image peak: $0.69 \mathrm{Jy} /$ beam. Contours: $1.5 \mathrm{mJy} /$ beam $\times$ $(-3,3,6,12,24,48,96,192,384)$. Beam FWHM: $1.18 \times 0.713$ (mas) at $-28.3^{\circ}, \mathrm{rms}=1.5 \mathrm{mJy} /$ beam

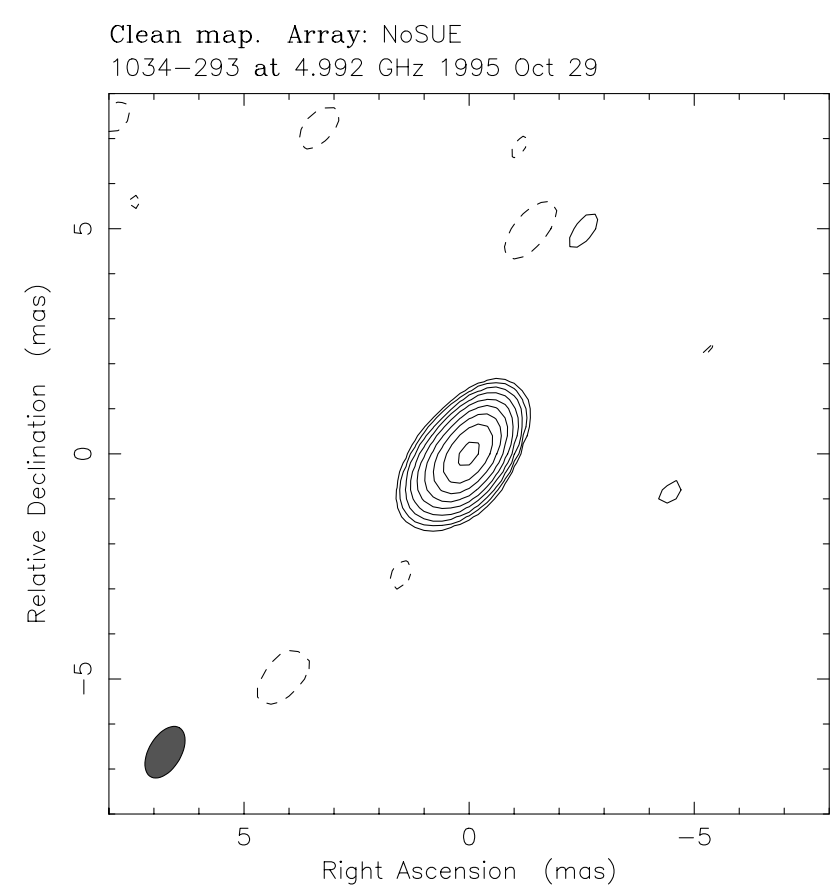

Fig. 10. Image peak: $0.91 \mathrm{Jy} /$ beam. Contours: $1 \mathrm{mJy} /$ beam $\times$ $(-3,3,6,12,24,48,96,192,384,768)$. Beam FWHM: $1.26 \times$ 0.72 (mas) at $-30.5^{\circ}, \mathrm{rms}=1 \mathrm{mJy} /$ beam 


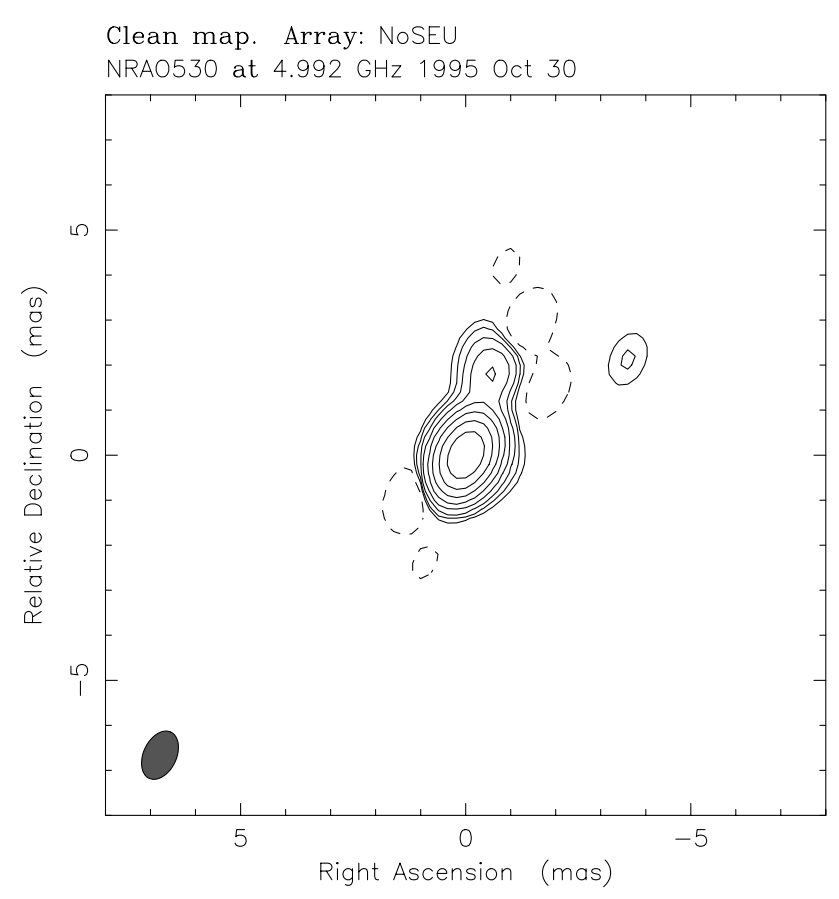

Fig. 11. Image peak: $2.95 \mathrm{Jy} /$ beam. Contours: $4 \mathrm{mJy} /$ beam $\times$ $(-3,3,6,12,24,48,96,192,384)$. Beam FWHM: $1.13 \times 0.73$ (mas) at $-25.2^{\circ}, \mathrm{rms}=4 \mathrm{mJy} / \mathrm{beam}$

Our VLBI image of the source (Fig. 9) shows a compact component. The structure can be fitted with one Gaussian component (Table 3). The flux density of the component is $0.73 \mathrm{Jy}$ which is only $75 \%$ of the total flux density. This suggests that extended emission may exist.

\subsection{Structural information for two more sources}

\section{$1034-293$}

1034-293 is a radio-selected blazar with redshift $z=0.312$. High optical polarization and X-ray emission were detected (Wills et al. 1992; Maraschi et al. 1995). Our image of the source shows an extended emission in $\mathrm{PA}=132^{\circ}$ (Fig. 10). The data can be fitted with two components with 1.63 mas separation. Because of the poor $(u, v)$ coverage and the lack of short baselines of our VLBI array, the extended structure was not so clearly imaged as in the VLBA images (Fey et al. 1996), where the source shows a core-jet morphology both at 2.3 and $8.5 \mathrm{GHz}$ in $\mathrm{PA}=140^{\circ}$. The flux density at $5 \mathrm{GHz}$ of the core decreased from 1.5 Jy (Shen et al. 1998) in 1992 to about 1 Jy in 1995 .

\section{0-130 (NRA0530)}

NRAO530 $(z=0.902)$ is a well known optically violent variable blazar, identified with a $\gamma$-ray source (Fichtel et al. 1994). It has been monitored at low frequency to look for variability (Ghosh et al. 1994; Bondi et al. 1994). Weak polarization was detected both at optical and radio
Table 5. Average correlated flux density information of 10 sources

\begin{tabular}{ccc|ccc}
\hline Source & $\begin{array}{c}S_{\mathrm{c}} \\
(\mathrm{Jy})\end{array}$ & $\begin{array}{c}\text { Baseline } \\
(\mathrm{M} \lambda)\end{array}$ & Source & $\begin{array}{c}S_{\mathrm{c}} \\
(\mathrm{Jy}) \\
(2)\end{array}$ & $\begin{array}{c}\text { Baseline } \\
(\mathrm{M} \lambda)\end{array}$ \\
$(1)$ & $(3)$ & $(1)$ & $(2)$ & $(3)$ \\
\hline $0008-264$ & 0.3 & $\sim 120$ & $0922+005$ & 0.7 & $40-150$ \\
$0108-079$ & 0.5 & $\sim 40$ & $1012+232$ & 0.4 & $40-100$ \\
$0256+075$ & 0.5 & $40-160$ & $1430-178$ & 0.4 & $\sim 100$ \\
$0338-214$ & 0.7 & $100-140$ & $2131-021$ & 1.4 & $100-140$ \\
$0528-250$ & 0.5 & $\sim 90$ & $2325-150$ & 0.6 & $100-140$ \\
\hline
\end{tabular}

bands. VLA observation at $1.4 \mathrm{GHz}$ showed an unresolved core and a second unresolved, possibly unrelated, component at about $11^{\prime \prime}$ in $\mathrm{PA}=270^{\circ}$ (Perley 1982).

Our image of 1730-130 shows a core-jet structure (Fig. 11). The jet is oriented in $\mathrm{PA}=-20^{\circ}$. The data can be fitted with two components separated by 2.0 mas, i.e. a strong component, possibly the core of the radio emission, with flux density $S=3.4$ Jy and a jet with $S=0.5 \mathrm{Jy}$. The jet orientation is roughly in agreement with the $1.7 \mathrm{GHz}$ image, which shows the structure extending out to 26 mas in $\mathrm{PA}=-7^{\circ}$ (Romney et al. 1984), but it is obviously different from the $5 \mathrm{GHz}$ VLBI image by Shen et al. (1997), where the position angle of the jet changes from $\mathrm{PA}=25^{\circ}$ at 1.3 mas to $\mathrm{PA}=19^{\circ}$ at 4.0 mas. The complex structural variations are difficult to be explained in terms of angular expansion. The flux density of the strongest component increased from 2.34 Jy in 1992 (Shen et al. 1997) to $3.4 \mathrm{Jy}$ in 1995. This may indicate an outburst of the birth of a new jet component. More observations of the source with better $(u, v)$ coverage are necessary to obtain the proper motion in the jet component and to study the relation of the emission mechanisms between radio and high energy radiation.

\subsection{Correlated flux density information of the remaining 10 sources}

Four of the remaining ten sources in our survey, i.e. $0256+075,0338-214,2131-021$, and 2325-150, were detected on most baselines with strong fringes, while the other six, 0008-264, 0108-079, 0528-250, 0922+005, $1012+232$, and 1430-178, were barely detected on some baselines, with poor fringes. The average (over baselines) correlated flux densities of the sources are presented in Table 5. Column 3 lists the spacing of detected baselines.

\section{Summary}

In this paper we have presented the results of 21 extragalactic radio sources of VLBI observation at $5 \mathrm{GHz}$. The VLBI images of eleven sources and the correlated flux density information of the remaining 10 sources are given. The superluminal nature of four sources (DA193, 
0420-014, 1334-127 and 2345-16) is confirmed and their physical parameters $(\delta, \gamma$ and $\phi)$ are derived. Lorentz factors in the range $3.5 \leq \gamma<10.4$ are found, in agreement with their blazar nature. Two new superluminal candidates $1504-166$ and $2243-123$ are found, and finally three sources $(1532+016,2320-035,2351-154)$ are imaged for the first epoch at VLBI resolution.

Acknowledgements. The authors are grateful to the staff of Medicina, Noto, Hartebeesthoek, Urumqi, and Shanghai radio telescopes for support during the VLBI observations, and the correlator and computer groups at Medicina for support of our project. XYH thanks IRA-CNR and JIVE/NFRA for their hospitality during his visit to Italy and the Netherlands. $\mathrm{XYH}$ is very grateful to Dr. L.I. Gurvits for his valuable comments. XYH is supported by the National Science Foundation of China, PAN DENG Plan, and Su- Shu Huang Astrophysics Research Foundation of Academia Sinica. The research has made use of the NASA/IPAC extragalactic database (NED) which is operated by the Jet Propulsion laboratory, Caltech, under contract with the National Aeronautics and Space Adminstration.

\section{References}

Antonucci R.R.J., Ulvestad J.S., 1985, ApJ 294, 158

Bajkova A.T., Pyatunina T.B., Finkelstein A.M., 1996, Commun. of the Institue of Applied Astronomy, Vol. 87

Bondi M., Padrielli L., Gregorini L., et al., 1994, A\&A 287, 390

Briggs D.S., Davis R.J., Conway J.E., et al., 1994, VLB ARRAY MEMO, No. 697A

Brinkmann W., Siebert J., Boller T., 1994, A\&A 281, 355

Browne I.W.A., Perley R.A., 1986, MNRAS 222, 149

Charlot P., 1990, A\&A 229, 51

Fey A.L., Clegg A.W., Fomalont E.B., 1996, ApJS 105, 299

Fichtel C.E., Bertsch D.L., Chiang J., et al., 1994, ApJS 94, 551

Ghisellini G., Padovani P., Celotti A., et al., 1993, ApJ 407, 65

Ghosh T., Gopal-Krishna, Rao A.P., 1994, A\&AS 106, 29

Guijosa A., Daly R.A., 1996, ApJ 461, 600

Henstock D.R., Browne I.W.A., Wilkinson P.N., et al., 1995, ApJS 100, 1

Hewitt A., Burbridge G., 1993, ApJS 87, 451

Impey C.D., Neugebauer G., 1988, AJ 95, 307

Impey C.D., Tapia S., 1990, ApJ 354, 124

Krichbaum T.P., Standke K.J., Witzel A., et al., 1994, in Proc. 2nd EVN/JIVE Symp., Kus A.J., Schilizzi R.T., Borkowski K.M., Gurvits L.I. (eds.). Torun Radio Astronomy Observatory Press. Poland, p. 47

Madau P., Ghisellini G., Persic M., 1987, MNRAS 244, 257

Maisack M., Wood K.S., Gruber D.E., 1994, A\&A 284, 28

Maraschi L., Ghisellini G., Celotti A., 1992, ApJ 397, L5

Maraschi L., Ciapi A., Fossati G., et al., 1995, ApJ 443, 29

Marscher A.P., 1987, in Superluminal Radio Sources, Zensus J.A., Pearson T.J. (eds.). Cambridge University Press, New York, p. 280
Marscher A.P., Broderick J.J., 1981, ApJ 249, 406

Mc Adam W.B., White G.L., 1983, MNRAS 203, 317

McEwan N.J., Browne I.W.A., Crowther J.H., 1975, MNRAS 80,1

Morabito D.D., Niell A.E., Preston R.A., et al., 1986, AJ 91, 1038

Morganti R., Killeen N.E.B., Tadhunter C.N., 1993, MNRAS 263,1023

Neff S.G., Hutchings J.B., 1990 AJ 100, 1441

Padrielli L., Romney J.D., Bartel N., et al., 1986, A\&A 165, 53

Pearson T.J., Readhead A.C.S., 1981, ApJ 248, 61

Pearson T.J., Readhead A.C.S., 1988, ApJ 328, 114

Pearson T.J., Zensus J.A., 1987, in Superluminal Radio Sources, Zensus J.A., Pearson T.J. (eds.). Cambridge University Press, New York, p. 1

Perley R.A., 1982, AJ 87, 859

Polatidis A.G., Wilkinson P.N., Xu W., et al., 1995, ApJS 98, 1

Preston R.A., Morabito D.D., Jauncey D.L., 1983, ApJ 269, 387

Preston R.A., Morabito D.D., Williams J.G., et al., 1985, AJ 90, 1599

Punsly B., 1995, AJ 109, 1555

Readhead A.C.S., 1994, ApJ 426, 51

Robertson D.S., Carter W.E., Ray J.R., et al., 1993, AJ 105, 353

Romney J., Padrielli L., Bartel N., et al., 1984, A\&A 135, 289

Schilizzi R.T., Shaver P.A., 1981, A\&A 96, 365

Scott M.A., Readhead A.C.S., 1997, MNRAS 180, 539

Shen Z.-Q., Wan T.-S., Moran J.M., et al., 1997, AJ 114, 1999

Shen Z.-Q., Wan T.-S., Moran J.M., et al., 1998, AJ 115, 1375

Shepherd M.C., Person T.J., Taylor G.B., 1995, BAAS 27,903

Spangler S.R., Mutel R.L., Benson J.M., 1983, ApJ 271, 44

Stevens J.A., Litchfield S.J., Robson E.I., et al., 1995, MNRAS 275,1146

Taylor G.B., Vermeulen R.C., Pearson T.J., et al., 1994, ApJS 95,345

Thakkar D.D., Xu W., Readhead A.C.S., et al., 1995, ApJS 98,33

Tornikoski M., Valtaoja E., Terasranta H., et al., 1996, A\&AS 116,157

Vermeulen R.C., Cohen M.H., 1994, ApJ 430, 467

Vermeulen R.C., 1996, in Extragalactic Radio Sources, Ekers R., Fanti C., Padrielli L. (eds.). Kluwer, p. 57

Veron-Cetty M.P., Veron P., 1995, ESO Scientific Report No. 17

Wagner S.J., Camenzind M., Dreissigacker O., et al., 1995, A\&A 298, 688

Wehrle A.E., Cohen M.H., Unwin S.C., et al., 1992, ApJ 391, 589

Wills B.J., Wills D., Breger M., et al., 1992, ApJ 398, 454

Wright A.E., Otrupcek R.E., Parkes Catalogue 1990, Australia Telescope National Facility

Xu W., Readhead A.C.S., Pearson T.J., et al., 1995, ApJS 99, 297 